\title{
Defective interfering virus protects elderly mice from influenza
}

\author{
Paul D Scott, Bo Meng, Anthony C Marriott, Andrew J Easton and Nigel J Dimmock
}

\begin{abstract}
Background: We have identified and characterised a defective-interfering (DI) influenza A virus particles containing a highly deleted segment 1 RNA that has broad-spectrum antiviral activity. In young adult mice it exerts protection against several different subtypes of influenza A virus (defined here as homologous or genetically compatible protection) and against a paramyxovirus and an influenza B virus (heterologous or genetically unrelated protection). Homologous protection is mediated by replication competition between the deleted and full-length genomes, and heterologous protection occurs through stimulation of innate immunity, especially interferon type I.

Methods: A single dose of the protective DI virus was administered intranasally to elderly mice at $-7,-1$ and +1 days relative to intranasal challenge with influenza A virus.

Results: A single dose of the DI virus given 1 or 7 days protected elderly mice, reducing a severe, sometimes fatal disease to a subclinical or mild infection. In contrast, all members of control groups treated with inactivated DI virus before challenge became extremely ill and most died. Despite the subclinical/mild nature of their infection, protected mice developed solid immunity to a second infectious challenge.
\end{abstract}

Conclusions: The defective interfering virus is effective in preventing severe influenza A in elderly mice and may offer a new approach to protection of the human population.

Keywords: elderly defective-interfering virus, geriatric, influenza, mice, treatment

\section{Background}

As a result of improved standards in the home, workplace, nutrition, and healthcare, the elderly are becoming an ever increasing proportion of the world's population. Yet because of waning immune competence and social factors, such as increased risk of infection in the communities in which many of them live, the elderly are vulnerable to a variety of infections, and particularly those that cause respiratory disease [1-4]. Elderly people exhibit a range of immune deficiencies that can be manifested in many facets of the immune response including $\mathrm{T}$ cell activation, $\mathrm{CD} 4^{+}$and/or $\mathrm{CD} 8^{+} \mathrm{T}$ cell activity, cytotoxic $\mathrm{T}$ cell activity, IL-2 secretion, antibody production and/or avidity, dendritic cells and type I interferon production [2,5-15]

Many of the causative agents of the most important respiratory diseases are viral, and amongst these influenza viruses and human respiratory syncytial virus

\footnotetext{
* Correspondence: n.j.dimmock@warwick.ac.uk

School of Life Sciences, University of Warwick, Coventry CV4 7AL, UK
}

(HRSV) are pre-eminent [16,17]. These agents are highly contagious and cause outbreaks in domestic communities with a high level of morbidity and often mortality. Since 1977 two subtypes of influenza A virus (H1N1 and H3N2) and an influenza B virus have been responsible for seasonal influenza. Pandemic 2009 H1N1 influenza entered the mix in April 2009 and appears to have replaced seasonal $\mathrm{H} 1 \mathrm{~N} 1$ virus. The surface haemagglutinin and neuraminidase antigens of these viruses evolve continuously so that immunity gained from infection becomes redundant after approximately 4 years, leaving individuals susceptible to further infection [18]. Thus the seasonal vaccine has to be formulated to match as far as possible the influenza A and B virus strains that are forecast to be circulating in 6 months' time. Efficacy of the vaccine depends, inter alia, on immune competence which declines in old age, and on providing the vaccination several weeks before the virus is encountered to allow the immune response time to mature [19-21]. However, a recent meta-analysis has questioned
C Biomed Central

() 2011 Scott et al; licensee BioMed Central Ltd. This is an Open Access article distributed under the terms of the Creative Commons Attribution License (http://creativecommons.org/licenses/by/2.0), which permits unrestricted use, distribution, and reproduction in any medium, provided the original work is properly cited. 
the efficacy of influenza vaccines in the over-65s [22]. The influenza antivirals Tamiflu and Relenza that are now available are most effective when given before or as soon as possible after infection, but solid evidence for their usefulness and safety in the elderly has not been found [23]. Resistance to Tamiflu, the more widely used antiviral, is already widespread in seasonal H1N1 virus [24-26].

In attempting to tackle respiratory disease we have pioneered a novel approach that exploits a naturally occurring influenza $\mathrm{A}$ antiviral that is made by the virus itself. This is a specific defective-interfering (DI) virus that confers protection from infection in vivo. Its active principle is a highly deleted version of the viral genomic RNA which acts to inhibit productive infection [27-31]. Influenza (Orthomyxoviridae) DI viruses were the first to be recognised [32], and have been studied extensively $[30,33]$. However analysis is difficult as natural DI preparations contain many different DI sequences. In part this is because its single-stranded, negative sense RNA genome exists as 8 discrete segments and DI RNAs can probably arise from any segment. While they are formed mainly from the three largest virion RNAs, any one virion RNA can give rise to many different DI RNA sequences through variably located central deletions $[34,35]$. We solved this heterogeneity problem by producing cloned viruses that contain one major species of DI RNA, and have characterized one DI virus, containing the 244 DI RNA derived from virion segment 1 of A/ Puerto Rico/8/34 (PR8, H1N1), that is particularly active in protecting mice from a variety of different influenza A virus subtypes [36]. We call DI viruses that have demonstrable in vivo activity 'protecting viruses' [37]. Our protecting 244 RNA is encapsidated into authentic influenza virus particles, and these target the protecting RNA to cells that can be potentially infected by influenza virus.

Thus far we have established the efficacy of protecting virus by infecting young adult mice (approximately 5 weeks-old) representing several different inbred strains. Here we have investigated the antiviral efficacy of protecting virus in elderly (18-month-old) mice. Such animals have a range of age-related immune deficiencies that parallel those found in people $[6,10,12,38-44]$. The data show that elderly mice are not only protected against a strong influenza type A challenge but also develop immunity to reinfection with the same challenge virus.

\section{Methods}

\section{Viruses}

Protecting virus 244/PR8 originally arose spontaneously after transfection of $293 \mathrm{~T}$ cells with an infectious set of A/PR/8/34 plasmids [36]. 244/PR8 was amplified sequentially in MDCK cells and embryonated chicken's eggs, and purified by differential centrifugation through sucrose. Preparations were standardized at $2 \times 10^{5}$ $\mathrm{HAU} / \mathrm{ml}$ and stored in liquid nitrogen. DI RNA 244 comprises 395 nucleotides and is derived from segment 1 RNA of PR8 by a single central deletion. Segment 1 encodes PB2, a component of the virion polymerase. 244 DI RNA comprises nucleotides 1-244 and 21912341 of the A/PR8 minus-sense segment 1 RNA, and retains the original termini and sequences essential for replication and encapsidation. Analysis by RT-PCR with primers specific for genome segment 1 showed that the 244 RNA was the major defective RNA present. Helper virus infectivity was eliminated by irradiating with UV for 40 seconds at $253.7 \mathrm{~nm}$. This had little effect on the DI RNA because of its small UV-target size (395 nt) compared with the infectious viral genome (13,600 nt). Longer UV irradiation (8 minutes) destroys the protecting RNA but does not affect viral HA or neuraminidase (NA) activities [36], and provides a control for possible immune system-stimulation by antigen or receptorblocking by the virus proteins [36]. The challenge virus, influenza A/WSN/40, was grown in embryonated chicken's eggs. The infectivity of clarified allantoic fluid was titrated in MDCK cells as focus-forming units (FFU). The dose required to cause disease in elderly mice was $4.3 \times 10^{3} \mathrm{FFU}$.

\section{Mice}

We used inbred $\mathrm{C} 3 \mathrm{H} / \mathrm{He}-\mathrm{mg}$ mice of both sexes which had once been part of a colony breeding programme. These were approximately 18 months of age with a mean weight of $45 \mathrm{~g}$ (range 36-52 g) at the time of inoculation. Compared with young adults they had increased body fat and thinner fur, were more poorly groomed, and considerably reduced activity levels, demonstrating the general demeanour of an elderly animal. Males were housed individually and most of the females as pairs, all in opaque plastic containers with a transparent lid. They thrived best in relatively small cages $(35 \times 14 \times 13 \mathrm{~cm})$ and were provided with food and water ad lib, paper bedding and cardboard tubes for recreation. Mice were treated with just one dose of protecting virus at the start of the study period. All inoculations were given as an intranasal drop to both nares under light ether anaesthesia. The dose of protecting virus comprised $12 \mu \mathrm{g}$ virus protein. This relatively large dose [36] was chosen as insufficient elderly mice were available to permit titration. Individual mice were assessed clinically according to a scheme where each mouse is assigned a value from 1 to 5 according to increasing severity of disease, where 1 is well and 5 is dead, as detailed previously, and by mean group weight, on a daily basis [36]. All experiments were approved by 
the University of Warwick Ethics Committee and conform to the licence requirements of the UK Home Office.

\section{Results}

Protection by protecting virus 1 day before infectious challenge

Mice were inoculated intranasally with a single dose of DI virus one day before intranasal infection with WSN. Figure 1a shows the clinical picture: mice given protecting virus alone or diluent (mock infected, data not shown) remained completely well. Those given UV-inactivated protecting virus before the infectious challenge became ill on day 5 , progressing to serious disease that peaked on days $7-8$. Most ( $67 \%$ or $4 / 6)$ were dead by day 8 . In contrast, mice treated with active $244 /$ PR 8 before infection were all protected. Sixty percent (3/5) were protected completely, while $2 / 5$ developed a mild illness, peaking on day 9 and from which they recovered completely. Comparison between the groups treated with active or inactive DI virus showed a highly significant statistical difference in the severity of disease $(\mathrm{p}=$ 0.0043) with a one tailed Mann-Whitney U test. Figure $1 \mathrm{~b}$ shows that body weight in both $244 / \mathrm{PR} 8$ alone controls oscillated around the zero mark, as did mock infected controls (not shown). This contrasts with young adults where there is a steady weight gain. Mice given inactivated 244/PR8 before challenge lost weight




at least 3 days ahead of clinical disease. Mice treated with 244/PR8 before infection showed only a small and transient weight loss, peaking at a maximum of $7 \%$ weight loss on days 9-10. They then regained the weight they had lost.

Having successfully protected elderly mice from acute influenza, we then determined their immune status. Animals that had received the active protecting virus and had survived the initial challenge infection were given a second WSN challenge at 3 weeks after the first infection to determine their immune status. This WSN dose $\left(2.1 \times 10^{6} \mathrm{FFU} /\right.$ mouse $)$ was 500 -fold greater than the first dose and is high enough to overcome protection afforded by the initial 244/PR8 treatment. Thus any resulting protection is probably due to acquired immunity. Figure 1 (panels c and d) show that mice protected from the initial WSN challenge by administration of 244/PR8 (from Figure 1 (panels a and b)) showed no sign of clinical disease on rechallenge, whereas mice from the same experiment given 244/PR8 alone as a first treatment were all dead 6 days after challenge (Figure 1c). A virus control of naïve 5-week-old mice was also included to verify the potency of the challenge virus and these all died. Clearly 244/PR8 on its own did not stimulate a protective immunity. Since 244/PR8 cannot protect against this high dose of WSN, we conclude that protected mice had developed an adaptive immune response. Weight change data (Figure 1d) corroborated the clinical data with the 244/PR8-protected mice from Figure 1 (panels a and b) showing no significant weight change.

\section{Protection by protecting virus given 7 days before, and 1 day after, infectious challenge}

Mice were given a single intranasal dose of 244/PR8 at 7 days and 1 day prior to infection with WSN, and at 1 day after infection. Controls received the same dose of inactivated 244/PR8. Figure 2 (panels a and b) show that groups given inactivated 244/PR8 and WSN in the various timed combinations or WSN alone all became seriously ill, lost a major amount of weight, and suffered $40 \%$ death. In contrast, $80 \%$ (4/5) mice given 244/PR8 at 7 days before infection were completely protected with no significant weight loss. The non-protected mouse showed no delay in the onset of illness and no amelioration of clinical signs, suggesting that it may not have received a full dose of $244 / \mathrm{PR} 8$. The positive control group given 244/PR8 1 day before WSN were solidly protected (as in Figure 1 (panels a and b)) with only delayed slight, short lived clinical signs on days 7 and 8 in 2 of 5 mice, consistent with the data in Figure 1 panels a and b (data not shown). Comparison between the groups treated with active or inactive DI virus showed a highly significant statistical difference in the severity of disease $(\mathrm{p}=0.0278)$ with a one tailed MannWhitney U test. 244/PR8 given one day after infection did not protect $(\mathrm{p}=0.3452$ comparing active and inactive DI treated mice and $p=0.4206$ comparing active DI treated mice with infected untreated mice). Surviving mice were challenged with the very high dose of WSN as above to determine their immune status. Figure 2 (panels c and d) show that mice that survived the first infection through treatment with 244/PR 8 at 1 day before infection were all solidly protected, as were most ( $75 \%$ or $3 / 4$ ) of the group that originally received $244 /$ PR8 at 7 days before infection. However, in such elderly mice it is expected that some mortality is associated with the age of the animals rather than infection. Agematched control mice lost weight, became ill and died.

\section{Discussion and conclusions}

We have previously shown that protecting virus prevents influenza in young adult mice. Protecting virus has two different modes of action. The observed crossinfluenza A subtype antiviral activity of DI virus 244/ PR8, coupled with its replication dependence on infectious virus and the common genetic system of all influenza A viruses, suggest that it acts at the level of genome competition [36]. Thus, it is likely that protecting virus can act against all influenza A viruses regardless of antigenicity, and indeed protecting virus prevents acute influenza A disease in SCID mice that have no adaptive immune response (submitted for publication). In addition, knock-out mice that have no functioning interferon type I response are also protected showing that this response is not essential for protection against challenge with homologous influenza A virus [45]. However, protecting virus stimulates interferon type I, and we believe this is responsible for heterologous protection in vivo from infection with pneumonia virus of mice (PVM), the acknowledged model for HRSV infection, and also influenza B virus. The importance of interferon type I in combatting these infections is shown by extensive reduction in protection against PVM and influenza $B$ in mice lacking the interferon type I receptor [45]. In view of the potential role of the immune system in the mode of action of the protecting virus it is important to assess the efficacy of protecting virus in older mice which have a range of immune deficiencies reflecting those seen in the high risk elderly human population.

The data above demonstrate that a single dose of prophylactic 244/PR8 given up to 7 days prior to infection was highly effective in protecting elderly mice from a severe influenza challenge, and that most of the surviving mice were immune to reinfection. Protection at both levels operates despite the multiplicity of age-related immune deficiencies found in such elderly mice (see above). However, a single dose post-infection treatment was less 


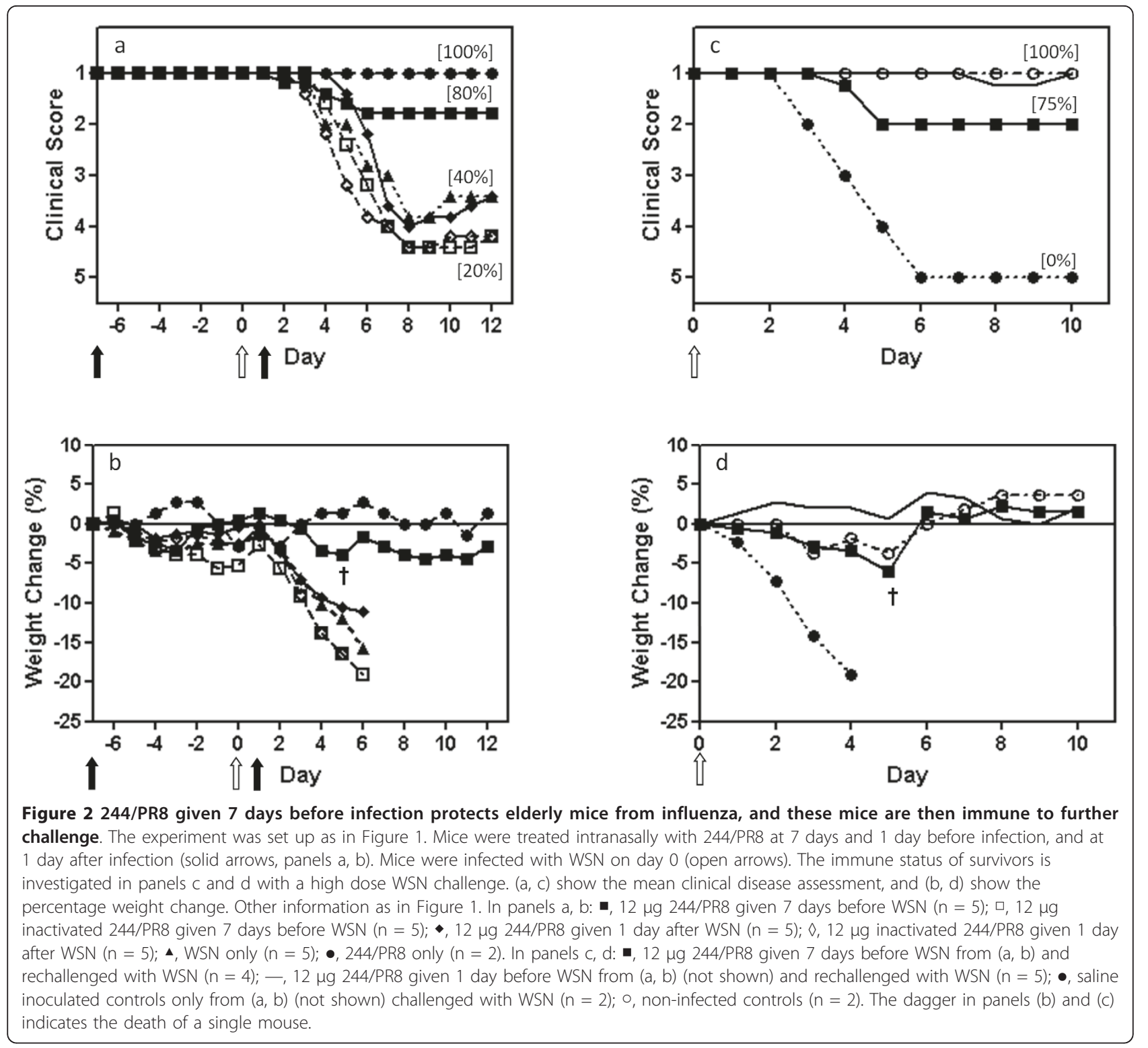

effective than in young adult mice [36] suggesting that elderly mice are less able to respond to this type of therapy. We have not yet explored the nature of the resistance to reinfection in elderly mice, but parallel experiments show that treatment of young adult mice with protecting virus prevents the presentation of any sign of disease, but permits a reduced level of productive influenza lung infection. The residual level of virus replication is sufficient to elicit an immune response which can protect against subsequent challenge with an antigenically related virus [36]. It seems likely therefore that there is also enough infection in elderly mice treated with protecting virus to stimulate a virus-specific B cell- and T cell-mediated immunity. In conclusion, both elderly and young adult mice are capable of benefitting from treatment with protecting virus.
Parallels with the elderly immunodeficient people suggest that protecting virus might be a useful additional weapon in the war against influenza.

\section{Acknowledgements}

We thank the Wellcome Trust and the UK Medical Research Council for grants awarded to NJD, AJE and ACM, and Samantha Dixon and staff for animals and their care.

\section{Authors' contributions}

NJD initiated the projected and carried it out with PDS. BM characterized the 244/PR8 and with ACM and AJE critiqued the work and contributed to the writing. This work has been read and approved by all authors.

\section{Competing interests}

NJD and AJE hold patents relating to DI influenza virus. Otherwise the authors declare that they have no competing interests. 
Received: 19 January 2011 Accepted: 9 May 2011 Published: 9 May 2011

\section{References}

1. Treanor JJ, Falsey A: Respiratory viral infections in the elderly. Antiviral Research 1999, 44:79-102.

2. Webster RG: Immunity to influenza in the elderly. Vaccine 2000, 18:1686-1689.

3. Falsey A, Dallal G, Formica M, Andolina G, Hamer D, Leka L, Meydani S: Long-term care facilities: A cornucopia of viral pathogens. Journal of the American Geriatrics Society 2008, 56:1281-1285.

4. Yoshikawa TT, Norman DC: Infectious disease in the aging Towota, NJ: Humana Press; 2000.

5. Canaday D, Amponsah N, Jones L, Tisch D, Hornick T, Ramachandra L: Influenza-induced production of interferon- $a$ is defective in geriatric individuals. Journal of Clinical Immunology 2010, 30:373-383.

6. Fujihashi K, Koga T, McGhee JR: Mucosal vaccination and immune responses in the elderly. Vaccine 2000, 18:1675-1680.

7. Fulop T, Pawelec G, Castle S, Loeb M: Immunosenescence and vaccination in nursing home residents. Clinical Infectious Diseases 2009, 48:443-448.

8. Huang YP, Pechere JC, Michel M, Gauthey L, Loreto M, Curran JA, Michel JP: In vivo T cell activation, in vitro defective IL-2 secretion, and response to influenza vaccination in elderly women. Journal of Immunology 1992, 148:715-722.

9. Joshi SR, Shaw AC, Quagliarello VJ: Pandemic influenza H1N1 2009, innate immunity, and the impact of immunosenescence on influenza vaccine. Yale Journal of Biology and Medicine 2009, 82:143-151.

10. Kovaiou RD, Grubeck-Loebenstein B: Age-associated changes within CD4+ T cells. Immunology Letters 2006, 107:8-14.

11. Mbawuike IN, Lange AR, Couch RB: Diminished influenza A virus-specific MHC class I-restricted cytotoxic T lymphocyte activity among elderly persons. Viral Immunology 1993, 6:55-64.

12. Reis e Sousa C: Dendritic cells in a mature age. Nature Reviews in Immunology 2007, 6:476-483.

13. Romero-Steiner S, Musher DM, Cetron MS, Pais LB, Groover JE, Fiore AE, Plikaytis BD, Carlone GM: Reduction in functional antibody activity against Streptococcus pneumoniae in vaccinated elderly individuals highly correlates with decreased IgG antibody avidity. Clinical and Infectious Disease 1999, 29:281-288.

14. Vallejo AN: Age-dependent alterations of the $\mathrm{T}$ cell repertoire and functional diversity of T cells of the aged. Immunological Research 2007, 36:221-228.

15. Whitley RJ, Monto AS: Prevention and treatment of influenza in high-risk groups: children, pregnant women, immunocompromised hosts, and nursing home residents. Journal of Infectious Diseases 2006, 194(S2): S133-S138.

16. Elliot A, Fleming D: Influenza and respiratory syncytial virus in the elderly. Expert Review of Vaccines 2008, 7:249-258.

17. Cox NJ, Subbarao K: Influenza. The Lancet 1999, 354:1277-1282.

18. Wilson IA, Cox NJ: Structural basis of immune recognition of influenza virus hemagglutinin. Annual Review of Immunology 1990, 8:737-771

19. Gross PA, Hermogenes A, Sacks HS, Lau J, Levandowski RA: The efficacy of influenza vaccine in elderly persons. A meta-analysis and review of the literature. Annals of Internal Medicine 1995, 123:518-527.

20. Jefferson T, Rivetti D, Rudin M, Di Pietrantonj C, Demicheli V: Efficacy and effectiveness of influenza vaccines in elderly people: a systematic review. Lancet 2005, 366:1165-1174

21. Goodwin K, Viboud C, Simonsen L: Antibody response to influenza vaccination in the elderly: a quantitative review. Vaccine 2006, 24:1159-1169

22. Jefferson T, Di Pietrantonj C, Al-Ansary LA, Ferroni E, Thorning S, Thomas RE: Vaccines for preventing influenza in the elderly. Cochrane Database of Systematic Reviews 2010, 2(2):CD004876.

23. Jefferson $T$, Jones $M$, Doshi $P$, Del Mar C, Dooley L, Foxlee R: Neuraminidase inhibitors for preventing and treating influenza in healthy adults. Cochrane Database of Systematic Reviews, Issue 2, article number CD001265 2010.

24. McKimm-Breschkin JL, Selleck PW, Usman TB, Johnson MA: Influenza (H5N1) with decreased oseltamivir sensitivity, Indonesia. Emerging Infectious Diseases 2007, 13:1354-1357.

25. Monto A: Implications of antiviral resistance of influenza viruses. Clinical Infectious Diseases 2009, 48:397-399.
26. Aoki FY, Boivin G, Roberts N: Influenza virus susceptibility and resistance to oseltamivir. Antiviral Therapy 2007, 12:603-616.

27. Holland JJ: Defective viral genomes. In Virology. Volume 1.. 2 edition. Edited by: Fields BN, Knipe DM. New York: Raven Press; 1990:151-165.

28. Holland JJ: Generation and replication of defective viral genomes. In Virology.. 2 edition. Edited by: Fields BN, Knipe DM. New York: Raven Press; 1990:77-99.

29. Huang AS, Baltimore D: Defective viral particles and viral disease processes. Nature (London) 1970, 226:325-327.

30. Nayak DP, Chambers TM, Akkina RM: Structure of defective-interfering RNAs of influenza virus and their role in interference. In The Influenza Viruses. Edited by: Krug RM. New York: Plenum Press; 1989:269-317.

31. Perrault J: Origin and replication of defective interfering particles. Current Topics in Microbiology and Immunology 1981, 93:151-207.

32. von Magnus P: Incomplete forms of influenza virus. Advances in Virus Research 1954, 21:59-79.

33. Nayak DP, Chambers TM, Akkina RK: Defective-interfering (DI) RNAs of influenza viruses: origin, structure, expression and interference. Current Topics in Microbiology and Immunology 1985, 114:103-151.

34. Jennings PA, Finch JT, Winter G, Robertson JS: Does the higher order of the influenza virus ribonucleoprotein guide sequence rearrangements in influenza viral RNA. Cell 1983, 34:619-627.

35. Duhaut SD, Dimmock NJ: Heterologous protection against a lethal human H1N1 influenza virus infection of mice by a H3N8 equine defective interfering virus: comparison of defective RNA sequences isolated from the DI inoculum and mouse lung. Virology 1998, 248:241-253.

36. Dimmock NJ, Rainsford EW, Scott PD, Marriott AC: Influenza virus protecting RNA: an effective prophylactic and therapeutic antiviral. Journal of Virology 2008, 82:8570-8578.

37. Marriott AC, Dimmock NJ: Defective interfering viruses and their potential as antiviral agents. Reviews in Medical Virology 2010, 20:51-62.

38. Toapanta FR, Ross TM: Impaired immune responses in the lungs of aged mice following influenza infection. Respiratory Research 2009, 10:112.

39. Wen ZY, Ye L, Gao YL, Pan L, Dong K, Bu ZG, Compans RW, Yang CL: Immunization by influenza virus-like particles protects aged mice against lethal influenza virus challenge. Antiviral Research 2009, 84:215-224.

40. Dong L, Mori I, Hossain MJ, Kimura Y: The senescence-accelerated mouse shows aging-related defects in cellular but not humoral imunity against influenza virus. Journal of Infectious Diseases 2000, 182:391-396.

41. Dong L, Mori I, Hossain MJ, Liu B, Kimura Y: An immunostimulatory oligodeooxynucleotide containing a cytidine-guanosine motif protects senescence-accelerated mice from lethal influenza virus by augmenting the T helper type 1 response. Journal of General Virology 2003, 84:1623-1628.

42. Effros RB, Walford RL: The immune response of aged mice to influenza: diminished T-cell proliferation, interleukin 2 production and cytotoxicity. Cellular Immunolology 1983, 81:298-305.

43. Po JL, Gardner EM, Anaraki F, Katsikis PD, Murasko DM: Age-associated decrease in virus-specific CD8+ T lymphocytes during primary influenza infection. Mechanisms of Ageing and Development 2002, 123:1167-1181.

44. Zheng B, Zhang Y, He H, Marinova E, Switzer K, Wansley D, Mbawuike I, Han S: Rectification of age-associated deficiency in cytotoxic $T$ cell response to influenza $\mathrm{A}$ virus by immunization with immune complexes. Journal of Immunology 2007, 179:6153-6159.

45. Easton AJ, Scott PD, Edworthy NL, Meng B, Marriott AC, Dimmock NJ: A novel broad-spectrum treatment for respiratory virus infections: influenza-based defective interfering virus provides protection against pneumovirus infection in vivo. Vaccine 2011, 29:2777-2784.

doi:10.1186/1743-422X-8-212

Cite this article as: Scott et al:: Defective interfering virus protects elderly mice from influenza. Virology Journal 2011 8:212. 